\title{
Accuracy of Immunodiagnostic Tests for Active Tuberculosis Using Single and Combined Results: A Multicenter TBNET-Study
}

\author{
Delia Goletti ${ }^{1 *}$, Carrara Stefania ${ }^{1}$, Ornella Butera ${ }^{1}$, Massimo Amicosante ${ }^{2}$, Martin Ernst ${ }^{3}$, Ilaria Sauzullo ${ }^{4}$, \\ Vincenzo Vullo ${ }^{4}$, Daniela Cirillo ${ }^{5}$, Emanuele Borroni ${ }^{5}$, Roumiana Markova ${ }^{6}$, Roumiana Drenska ${ }^{6}$, José \\ Dominguez ${ }^{7}$, Irene Latorre ${ }^{7}$, Claudio Angeletti ${ }^{8}$, Assunta Navarra ${ }^{8}$, Nicola Petrosillo ${ }^{9}$, Francesco Nicola \\ Lauria $^{8}$, Giuseppe Ippolito ${ }^{8}$, Giovanni Battista Migliori ${ }^{10}$, Christoph Lange ${ }^{3}$, Enrico Girardi ${ }^{8}$
}

1 Translational Research Unit, Department of Epidemiology and Preclinical Research, National Institute for Infectious Diseases (INMI) L. Spallanzani, IRCCS, Rome, Italy, 2 Department of Internal Medicine, University "Tor Vergata”, Rome, Italy, 3 Division of Clinical Infectious Diseases, Research Center Borstel, Borstel, Germany, 4 Infectious and Tropical Diseases Department, "La Sapienza" University, Rome, Italy, $\mathbf{5}$ Emerging Bacterial Pathogens Unit, "San Raffaele" Scientific Institute, Milan, Italy, 6 Department of Immunology and Allergology, National Center for Infectious and Parassitic Diseases, Sofia, Bulgaria, 7 Fundació Institut d'Investigació en Ciències de la Salut Germans Trias i Pujol, CIBER Enfermedades Respiratorias, Universitat Autònoma de Barcelona, Barcelona, Spain, 8 Clinical Epidemiology, Department of Epidemiology and Preclinical Research, INMI "L.Spallanzani", Rome, Italy, 9 Clinical Department, INMI "L.Spallanzani", Rome, Italy, 10 WHO Collaborating Centre for Tuberculosis and Lung Diseases, S. Maugeri Foundation, IRCCS, Tradate, Italy

\begin{abstract}
Background: The clinical application of IFN- $\gamma$ release assays (IGRAs) has recently improved the diagnosis of latent tuberculosis infection. In a multicenter study of the Tuberculosis Network European Trialsgroup (TBNET) we aimed to ascertain in routine clinical practice the accuracy of a novel assay using selected peptides encoded in the mycobacterial genomic region of difference (RD) 1 for the diagnosis of active tuberculosis in comparison with tuberculin skin test (TST), QuantiFERON-TB GOLD In-Tube (Cellestis Ltd., Carnegie, Australia) and T-SPOT.TB (Oxfordimmunotec, Abingdon, UK).

Principal Findings: 425 individuals from 6 different European centres were prospectively enrolled. We found that sensitivity of the novel test, TST, QuantiFERON-TB GOLD In-Tube and T-SPOT.TB was respectively $73.1 \%, 85.3 \%, 78.1 \%$, and $85.2 \%$; specificity was respectively $70.6 \%, 48.0 \%, 61.9 \%$ and $44.3 \%$; positive likelihood ratios were respectively $2.48,1.64,2.05$, and 1.53 ; negative likelihood ratios were respectively $0.38,0.31,0.35,0.33$. Sensitivity of TST combined with the novel test, QuantiFERON-TB GOLD In-Tube and T-SPOT.TB increased up to $92.4 \%, 97.7 \%$ and $97.1 \%$, respectively. The likelihood ratios of combined negative results of TST with, respectively, the novel test, QuantiFERON-TB GOLD In-Tube and T-SPOT.TB were 0.19, 0.07 and 0.10 .

Conclusions: The assay based on RD1 selected peptides has similar accuracy for active tuberculosis compared with TST and commercial IGRAs. Then, independently of the spectrum of antigens used in the assays to elicit mycobacterial specific immune responses, the novel test, IGRAs, and the TST do not allow an accurate identification of active tuberculosis in clinical practice. However, the combined use of the novel assay or commercial IGRAs with TST may allow exclusion of tuberculosis.
\end{abstract}

Citation: Goletti D, Stefania C, Butera O, Amicosante M, Ernst M, et al. (2008) Accuracy of Immunodiagnostic Tests for Active Tuberculosis Using Single and Combined Results: A Multicenter TBNET-Study. PLoS ONE 3(10): e3417. doi:10.1371/journal.pone.0003417

Editor: Madhukar Pai, McGill University, Canada

Received July 24, 2008; Accepted September 22, 2008; Published October 15, 2008

Copyright: (C) 2008 Goletti et al. This is an open-access article distributed under the terms of the Creative Commons Attribution License, which permits unrestricted use, distribution, and reproduction in any medium, provided the original author and source are credited.

Funding: The paper was supported by grants from the Italian Ministry of Health (Progetto di Ricerca I.S.S. n. 06.76 and Ricerca Corrente INMI)

Competing Interests: DG, SC, MA and EG have a patent pending on T-cell assay based on RD1 selected peptides.

*E-mail: d.goletti@tiscali.it

\section{Introduction}

Tuberculosis control is based on the consequent use of preventive chemotherapy in individuals with latent tuberculosis infection (LTBI) who are at risk of developing active disease and on the rapid diagnosis and effective treatment of infectious cases [1-3]. While the identification of patients with active tuberculosis can rapidly be established by the detection of alcohol acid fast bacilli (AFB) on sputum smears, early diagnosis of infectious cases by sputum microscopy is only possible in approximately $50 \%$ of cases [4]. The sub-optimal performances of existing diagnostic tools [4], in terms of both speed and sensitivity, delayed diagnosis and, consequently, treatment of active tuberculosis.
The recent introduction of T-cell-based interferon (IFN)- $\gamma$ release assays (IGRAs), using antigens belonging to $M$. tuberculosis region of difference (RD) 1 (including early secreted antigenic target [ESAT]-6 and culture filtrate protein 10 [CFP]-10) represents a significant step towards improved LTBI diagnosis [5-9]. There is growing evidence that in low incidence settings both the commercial IGRAs currently available, the QuantiferonGOLD In-tube assay (Cellestis Ltd., Carnegie, Australia) and the T-SPOT.TB assay (Oxfordimmunotec, Abingdon, UK) are less affected by bacillus Calmette-Guerin (BCG) vaccination than the tuberculin skin test (TST) and that they are more specific and correlate better with exposure to an infected index case [10-13]. Although these commercial assays provide an accurate diagnosis of 
M. tuberculosis infection and detect active tuberculosis disease, they cannot discriminate between active tuberculosis and LTBI. Thus, further clinical workup is required to rule out active tuberculosis after a positive response to these tests.

Recently the design of a novel in vitro immune diagnostic enzyme-linked immunospot (ELISPOT) and whole blood ELISA (WBE) for IFN- $\gamma$ using multiepitopic peptides that are selected by computational analysis from CFP-10 and ESAT-6 as stimulating antigens has been reported [14]. It has been shown that the response to RD1 selected peptides can be detected in subjects with ongoing $M$. tuberculosis replication, such as during active tuberculosis and/or recent infection [15-17]. This response is mediated by $\mathrm{CD} 4^{+} \mathrm{T}$ effector cells, shown to undergo clonal expansion during $M$. tuberculosis replication, followed by a contraction phase after efficacious therapy culminating in the generation of $\mathrm{CD}^{+}$ memory T-cells $[18,19]$. These studies were conducted at one center in Italy, a country with a low tuberculosis incidence of $<10 / 100.000$ [20]. The aims of this multicenter study were: i) to evaluate whether this assay based on RDl selected peptides may help in providing evidence of active tuberculosis; ii) to compare the response to this novel assay with TST and the commercially available RD1 tests, individually and in combination for the diagnostic work-up of active tuberculosis [21].

\section{Materials and Methods}

\section{Study design}

Following obtaining of informed consent, patients with a clinical suspicion of tuberculosis (abnormal chest radiograph suggestive of tuberculosis and/or other signs and symptoms such as persistent cough, haemoptysis, weight loss, fever) were prospectively recruited at participating centers of the Tuberculosis Network European Trialsgroup (TBNET): Bulgaria (Department of Immunology and Allergology, National Center for Infectious and Parassitic Diseases, Sofia), Germany (Medical Clinic, Research Centre Borstel, Borstel), Italy (INMI and University La Sapienza, Rome; Scientific Institute San Raffaele, Milan) and Spain (Hospital Universitari Germans Trias i Pujol; Barcelona) between November 2005 and March 2008.

Patients underwent clinical and microbiological examinations including chest radiographs to confirm or exclude the diagnosis of tuberculosis. Briefly, 3 sequential respiratory expectorated or 2 induced sputum smears over the first 7 days following clinical evaluation were collected. AFB smear and culture (on both, Lowenstein-Jensen and Bactec MGIT, BD Biosciences Division, Sparks, Maryland, USA) were performed on each specimen. Additionally $M$. tuberculosis-specific RNA amplification was performed on specimens from patients with a high likelihood for tuberculosis in which examinations for AFB were negative [(GenProbe $^{\circledR}$ Amplified $^{\mathrm{TM}}$ Mycobacterium Tuberculosis Direct (MTD) Test, San Diego, CA, USA)]. TSTs were administered by the Mantoux method with bioequivalent 5 Tuberculin Units (Biocine, Chiron, Siena, Italy) or 2 Tuberculin Units (RT23, Statens Serum Institute, Copenhagen, Denmark) [22,23] or 5 Units of PPD Tuberculin Mammalian (BulBio-NCIPD, Sofia, Bulgaria). Indurations were measured 48-72 hours following tuberculin administration by the ballpoint technique. Individuals with an induration $\geq 10 \mathrm{~mm}$ [24] or in Bulgaria $\geq 15 \mathrm{~mm}$ [25-26] for those with past BCG vaccination were classified as TST-positive [22].

For extra-pulmonary tuberculosis, M. tuberculosis-specific RNA amplification (MTD Test) and/or nucleic acid amplification test (NAT) for $M$. tuberculosis-specific DNA based on a commercial test (BD ProbeTec ET system; BD Diagnostic Systems, Sparks, MD) or based on a homemade version developed from the literature
[27] was performed on biopsy specimens and/or biological fluids; moreover histology and AFB staining were performed on biopsies.

Enrolled patients were classified as "confirmed tuberculosis" if the diagnosis was based: i) in those with pulmonary tuberculosis by a positive culture for $M$. tuberculosis; ii) in those with extrapulmonary tuberculosis by a) positive $M$. tuberculosis-specific RNA amplification and/or $M$. tuberculosis-specific NAT from biological specimens or b) by histological pathological finding consistent with tuberculosis and presence of AFB in a tissue sample or c) by positive culture for M. tuberculosis in clinical samples (pleural fluid and abscesses). Conversely, patients were classified as "clinical tuberculosis" if the diagnosis was based on clinical and radiologic criteria (having excluded other disease) including appropriate response to anti-tuberculosis therapy.

We defined patients without tuberculosis as those admitted with a suspicion of active tuberculosis, who subsequently showed negative sputum for AFB smear and culture for M. tuberculosis with either a resolution of clinical symptoms and radiographic abnormalities after an antibiotic therapy not involving $M$. tuberculosis active drugs, or presenting a confirmed alternative diagnosis (e.g.: lung cancer).

Following admission, a 10-20 $\mathrm{ml}$ (depending on the center) heparin venous blood sample was drawn from all enrolled individuals. ELISPOT or WBE based on RD1 selected peptides was performed. In a subgroup of patients the test was done in parallel with the commercially available immune assays for tuberculosis. Clinicians were blinded to the results of in vitro assays and laboratory personnel were blinded to the status of the patient. The study was approved by the ethics committee at all the institutions in which the study was performed.

\section{RD1 selected peptides and stimuli used for cell cultures}

The selection of Human Leukocytes Antigens (HLA)-class II restricted epitopes of ESAT-6 and CFP-10 M. tuberculosis proteins was performed by quantitative implemented HLA peptide-binding motifs analysis as previously described for ESAT-6 [14,15]. Peptides were synthesized as free amino acid termini using Fmoc chemistry (ABI, Bergamo, Italy). Lyophilized peptides were diluted in DMSO at stock concentrations of $10 \mathrm{mg} / \mathrm{mL}$ for each peptide and stored at $-80^{\circ} \mathrm{C}$. RD1 selected peptides were used as follows: a pool of the two ESAT-6 peptides (at $10 \mu \mathrm{g} / \mathrm{mL}$ each), a pool of the three CFP10 peptides (at $2 \mu \mathrm{g} / \mathrm{mL}$ each). DMSO was used as negative control at $10 \mu \mathrm{g} / \mathrm{mL}$. As positive control we used Phytohemagglutinin (PHA) (Sigma, St Louis, MO, USA) at $5 \mu \mathrm{g} / \mathrm{mL}$. RDl selected peptides from the same batch were provided to the all centers with a detailed protocol. Four out of the 5 external centers received personal training from INMIs' laboratory personnel for at least 2days. Inter-site communication was present all over the performance of the study to solve any potential problem.

ELISPOT. $2.5 \times 10^{5}$ peripheral blood mononuclear cells (PBMC) were separated, washed twice and plated in the TSPOT.TB plates stimulated with or without RD1 selected peptides and PHA, as described above and previously [14,15]. Cell cultures were incubated overnight at $37^{\circ} \mathrm{C}$, with $5 \% \mathrm{CO} 2$. On the next morning, the cells were washed off, and the ELISPOT was developed following the manufacturer's instructions (Oxford Immunotec, UK). Spots were then counted by an automated ELISA-Spot assay video analysis system (AELVIS, Hannover, Germany). Evaluated spots had a size $>15 \mathrm{U}(1 \mathrm{U}=50 \mu \mathrm{m} 2)$. Indeterminate results were defined by values in the PHAstimulated samples below 34 spot-forming cells per million PBMC. The RDl selected peptide responses were scored as positive if above 34 spot-forming cells/million PBMC. This cutoff value was determined by constructing a receiver operator 
characteristic (ROG) curve by means of LABROG-1 software. To obtain the absolute value, the number of spot-forming cells in the negative controls was subtracted from the number of spot-forming cells in the stimulated cultures. Clinicians were blinded to the laboratory test results and laboratory personnel were blinded to the status of the patients.

WBE. Briefly, aliquots of $0.5 \mathrm{ml}$ per well of heparinized blood were seeded in a 48-well plate and stimulated with or without RD1 selected peptides and PHA, as described above. Samples were then incubated for 24 hours at $37^{\circ} \mathrm{C}$ in presence of $5 \% \mathrm{CO}_{2}$ when an amount of $100 \mu \mathrm{l}$ of plasma was harvested. IFN- $\gamma$ levels in culture supernatants were assessed by a commercially available kit (QuantiFERON-GMI kit, Cellestis). For the results scoring, a cut-off value of $0.7 \mathrm{IU} / \mathrm{mL}$ was chosen for all stimuli by constructing a ROG curve. Indeterminate results were defined by values in the PHA-stimulated samples below $0.7 \mathrm{IU} / \mathrm{mL}$.

\section{Commercially available assays}

T-SPOT.TB and QuantiFERON-TB GOLD In-Tube assays were performed and their results were scored as indicated by the manufacturers.

Statistical Analysis. The tests performance was evaluated by using categories of confirmed tuberculosis, clinical tuberculosis, and no active tuberculosis. Cases with indeterminate responses to in vitro assays were not included in the analysis. Sensitivity, specificity and likelihood ratios with their 95\% Confidence
Interval (CI), were computed for each test overall and according to the diagnostic categories and tuberculosis localization. Proportions were compared by using Fisher exact test and, for paired data, McNemar chi-square test. Sensitivities of 2 tests used in combination were obtained assuming that a positive result is given by a positive response to at least one assay.

Furthermore the accuracy of two tests used in combination was analyzed by computing the likelihood ratios, together with the distribution of subjects with and without active tuberculosis, according to the responses to the tests. Sensitivities and specificities of diagnostic tests were compared by using a logistic regression model with robust standard errors to account for the correlation between observations. Two-tailed $P$ values are reported.

\section{Results}

We consecutively enrolled 425 consenting adult patients from 6 different centres in Europe. Complete data were unavailable from 1 patient. Results from $11(2.5 \%)$ subjects were found to be indeterminate by in vitro assays based on RD1 selected peptides and/or QuantiFERON-TB GOLD In-Tube (Figure 1). Among them, 4 had active tuberculosis and 7 were without active tuberculosis. These patients were similar to those without indeterminate results in terms of age, sex, ethnicity, immune suppressive therapy intake and presence of comorbidity conditions (data not shown).

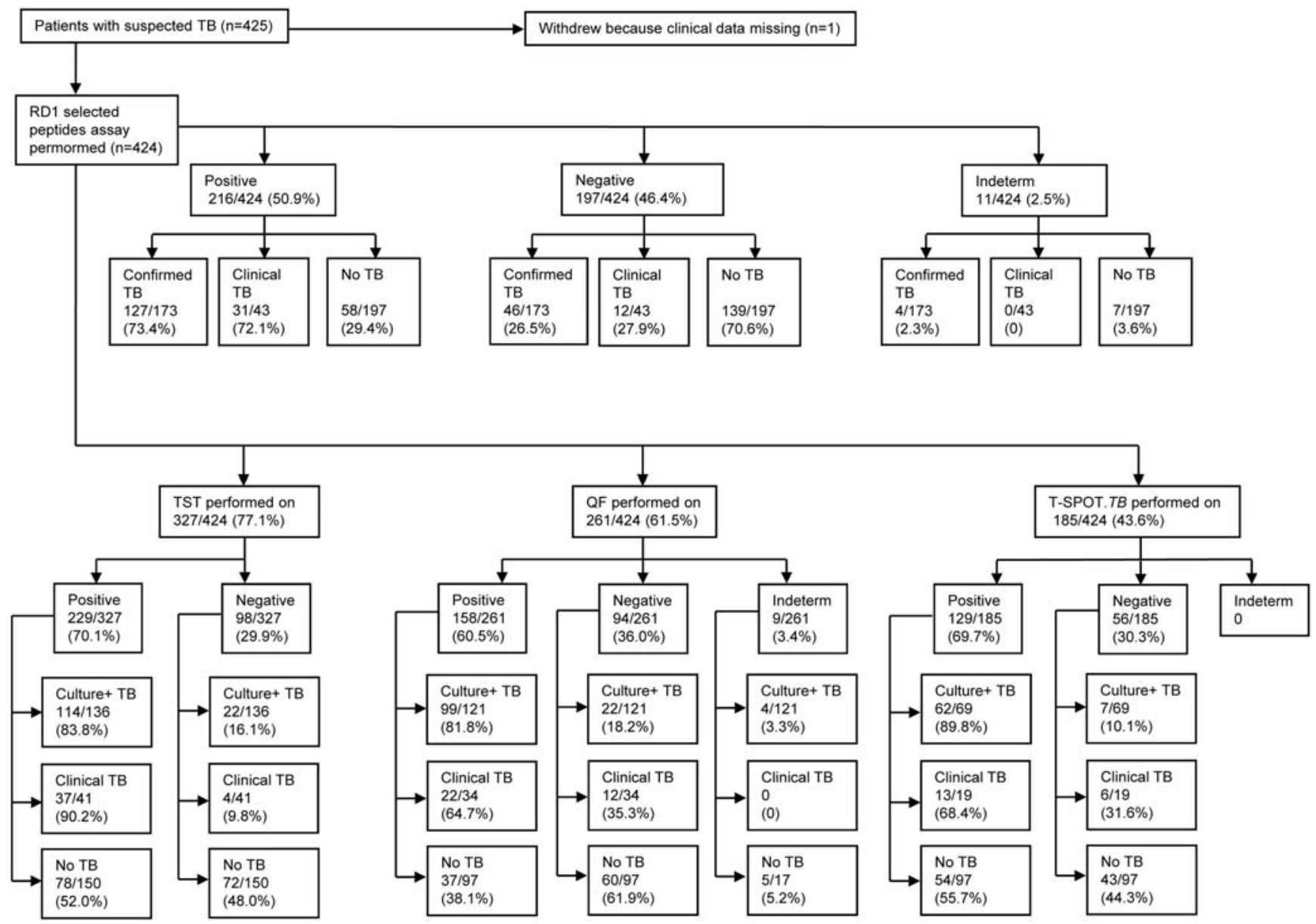

Figure 1. Study flow diagram. Abbreviations: TB: tuberculosis; RD: Region of Difference; Indeterm: indeterminate; TST: tuberculin skin test; QF: QuantiFERON-TB GOLD In-Tube.

doi:10.1371/journal.pone.0003417.g001 
Hereafter, the data analysis was performed only in the 413 samples with valid in vitro test results. Demographic characteristics of these subjects are shown in Table 1. Among them we classified 173 patients $(41.9 \%)$ as having confirmed tuberculosis and 43 $(10.4 \%)$ as having clinical tuberculosis. We excluded active tuberculosis in 197 patients $(47.7 \%)$. Based on localization site, $146(67.6 \%)$ were classified as having pulmonary tuberculosis, 56 $(25.9 \%)$ extrapulmonary tuberculosis and $14(6.5 \%)$ had both pulmonary and extrapulmonary localization (Table 2).

\section{Response to RD1 selected peptides assay and} comparison with the other tests

WBE and ELISPOT readouts significantly correlate for the detection of the responses to RD1 selected peptides. Evaluation of the response to RD1 selected peptides was performed by 2 different readouts, the ELISPOT and the WBE that we previously demonstrated to significantly correlate with each other [15]. Also in this study, 138 samples were run in parallel with a significant correlation (percentage of agreement: $80.4 \% ; p=0.0001)$. Moreover no differences were found in terms of detection of positive results in those with active tuberculosis among the patients from the different centers $(\mathrm{p}>0.5)$. Given the concordance of the results, the data were pooled together and analyzed as a whole.
Response to immunological tests for tuberculosis: assay based on RD1 selected peptides, TST, commercial IGRAs

For confirmed and clinical tuberculosis cases, diagnostic test sensitivities were 73.1\% (95\% CI, 66.7-78.9\%) with RD1 selected peptides test, $85.3 \%$ (95\% CI, 79.2-90.2\%) with TST, 78.1\% (95\% CI, 70.7-84.3\%) with QuantiFERON-TB GOLD In-Tube, and 85.2\% (CI, 76.1-91.9\%) with T-SPOT.TB (Table 2).

To investigate whether inclusion of patients with clinical tuberculosis in the analysis affected performance estimates, we re-estimated sensitivity by using only confirmed cases. Sensitivity remained stable and was $73.4 \%$ (CI, 66.2-79.8) with RD1 selected peptides test, $83.8 \%$ (CI, 76.5-89.6) with TST, 81.8\% (CI, 73.888.2\%) with QuantiFERON-TB GOLD In-Tube, and $89.9 \%$ (CI,80.2-95.8\%) with T-SPOT.TB (Table 2). No differences were found between the results obtained considering confirmed tuberculosis vs. clinical tuberculosis cases with the exception of T-SPOT.TB for which a higher proportion of positive results was observed for confirmed tuberculosis $(62 / 69)$ vs. clinical tuberculosis $(13 / 19, p=0.03)$. Results of immune responses were therefore evaluated for patients with confirmed and clinical tuberculosis pooled together, unless differently specified, and were defined as patients with active tuberculosis.

Among patients with active tuberculosis, the RDl selected peptides assay was less sensitive than TST (in the 170 patients with

Table 1. Demographic and clinical characteristics of the subjects enrolled in the study.

\begin{tabular}{|c|c|c|c|c|}
\hline & Confirmed TB & Clinical TB & No Active TB & Total \\
\hline & N. 173 (\%) & N. 43 (\%) & N. 197 (\%) & N. 413 (\%) \\
\hline Age years (median) & 34 & 36 & 48 & 40 \\
\hline \multicolumn{5}{|l|}{ Gender } \\
\hline Female & 69 (39.9) & $15(34.9)$ & $67(34.0)$ & $151(36.6)$ \\
\hline Male & $104(60.1)$ & $28(65.1)$ & $130(66.0)$ & $262(63.4)$ \\
\hline \multicolumn{5}{|l|}{ BCG } \\
\hline Yes & $105(60.6)$ & $7(16.2)$ & $54(27.4)$ & $166(46.0)$ \\
\hline No & $54(31.2)$ & $34(79.0)$ & $107(54.3)$ & $195(54.0)$ \\
\hline Unknown & $14(8.0)$ & $2(0.04)$ & $36(18.2)$ & $52(12.5)$ \\
\hline \multicolumn{5}{|l|}{ Origin } \\
\hline Africa & $29(16.8)$ & $6(14.0)$ & $11(5.6)$ & $46(11.1)$ \\
\hline Asia & $17(9.8)$ & $10(23.3)$ & $9(4.6)$ & $368.7)$ \\
\hline Eastern Europe & $66(38.2$ & $5(11.6)$ & $31(15.7)$ & $102(24.7)$ \\
\hline South America & $17(9.8)$ & 0 & $10(5.1)$ & $27(6.5)$ \\
\hline Western Europe & $44(25.4)$ & $22(51.2)$ & $136(69.0)$ & $202(48.9)$ \\
\hline \multicolumn{5}{|l|}{ Past TB } \\
\hline Yes & 0 & 0 & $27(13.7)$ & $27(6.5)$ \\
\hline No & $173(100)$ & $43(100)$ & $170(86.3)$ & $386(93.5)$ \\
\hline \multicolumn{5}{|c|}{ Immune suppressive therapy } \\
\hline Yes & $4(2.3)$ & $2(4.7)$ & $4(2.0)$ & $10(2.4)$ \\
\hline No & $169(97.7)$ & $41(95.3)$ & $193(98.0)$ & $403(97.6)$ \\
\hline \multicolumn{5}{|l|}{ HIV status } \\
\hline Yes & $3(1.7)$ & 0 & $4(2.0)$ & $7(1.7)$ \\
\hline No & $155(89.5)$ & $43(100)$ & $167(84.7)$ & $365(88.3)$ \\
\hline Unknown & $15(8.6)$ & 0 & $26(13.2)$ & $41(9.9)$ \\
\hline
\end{tabular}

\section{Abbreviations:}

TB: tuberculosis; BCG: Bacillus Calmette and Guerin; HIV: Human Immunodeficiency Virus.

doi:10.1371/journal.pone.0003417.t001 
Table 2. Accuracy for the diagnosis of active tuberculosis.

\begin{tabular}{|c|c|c|c|c|c|}
\hline \multicolumn{2}{|l|}{ Sensitivity* } & $\begin{array}{l}\text { RD1 selected } \\
\text { peptides test }\end{array}$ & TST & \multirow{3}{*}{$\begin{array}{l}\text { QuantiFERON-TB } \\
\text { GOLD In-Tube }\end{array}$} & \multirow{3}{*}{$\begin{array}{l}\text { T-SPOT.TB } \\
62 / 69(89.9)\end{array}$} \\
\hline & & \multicolumn{2}{|c|}{ Positive over total (\%) $[\mathrm{Cl}]$} & & \\
\hline \multirow{4}{*}{$\begin{array}{l}\text { According to diagnostic } \\
\text { criteria }\end{array}$} & Confirmed TB & $127 / 173(73.4)$ & 114/136 (83.8) & & \\
\hline & & {$[66.2-79.8]$} & {$[76.5-89.6]$} & {$[73.8-88.2]$} & {$[80.2-95.8]$} \\
\hline & Clinical TB & $31 / 43(72.1)$ & $37 / 41(90.2)$ & 22/34 (64.7) & 13/19 (68.4) \\
\hline & & {$[56.3-84.7]$} & [76.9-97.3] & {$[46.5-80.3]$} & {$[43.4-87.4]$} \\
\hline \multirow{6}{*}{$\begin{array}{l}\text { According to TB } \\
\text { localization }\end{array}$} & Pulmonary & $109 / 146(74.7)$ & 100/115 (87.0) & 88/107 (82.2) & $49 / 56(87.5)$ \\
\hline & & [66.8-81.5] & [79.4-92.5] & {$[73.7-89.0]$} & [75.9-94.8] \\
\hline & Extra-pulmonary & $40 / 56(71.4)$ & $41 / 47(89.4)$ & $26 / 39(66.7)$ & $17 / 23(73.9)$ \\
\hline & & {$[57.8-82.7]$} & [76.9-96.5] & [49.8-80.9] & [51.6-89.8] \\
\hline & $\begin{array}{l}\text { Pulmonary and extra- } \\
\text { pulmonary }\end{array}$ & $9 / 14(64.3)$ & $9 / 15(60.0)$ & $7 / 9(77.8)$ & 9/9 (100) \\
\hline & & {$[35.1-87.2]$} & {$[32.3-83.7]$} & {$[40.0-97.2]$} & [71.7-100.0] \\
\hline \multirow[t]{2}{*}{ Total } & & 158/216 (73.1) & 151/177 (85.3) & 121/155 (78.1) & $75 / 88(85.2)$ \\
\hline & & {$[66.7-78.9]$} & {$[79.2-90.2]$} & {$[70.7-84.3]$} & [76.1-91.9] \\
\hline \multicolumn{6}{|l|}{ Specificity** } \\
\hline & & 139/197 (70.6) & $72 / 150(48.0)$ & 60/97 (61.9) & 43/97 (44.3) \\
\hline & & {$[63.7-76.8]$} & {$[39.8-56.3]$} & {$[51.4-71.5]$} & {$[34.2-54.8]$} \\
\hline \multicolumn{6}{|l|}{ Positive likelihood ratio*** } \\
\hline & & 2.48 & 1.64 & 2.05 & 1.53 \\
\hline & & [1.97-3.1] & [1.39-1.94] & {$[1.57-2.67]$} & {$[1.26-1.87]$} \\
\hline \multicolumn{6}{|c|}{ Negative likelihood ratio*** } \\
\hline & & 0.38 & 0.31 & 0.35 & 0.33 \\
\hline & & {$[0.30-0.48]$} & {$[0.21-0.45]$} & {$[0.25-0.50]$} & {$[0.19-0.58]$} \\
\hline
\end{tabular}

* evaluated on the total number of positive results over the total number of patients with active tuberculosis disease.

** evaluated on the total number of negative results over the total number of patients without active tuberculosis disease.

evaluated on the total number of tuberculosis cases (confirmed and clinical tuberculosis).

Abbreviations:

TB: tuberculosis; RD: region of difference; TST: tuberculin skin test; $\mathrm{Cl}$ : confidence interval.

doi:10.1371/journal.pone.0003417.t002

results from both tests) $(\mathrm{p}=0.004)$ and T-SPOT.TB (in the 88 patients with results from both tests) $(p=0.008)$, but not the QuantiFERON-TB GOLD In-Tube (in the 154 patients with results from both tests) $(p=0.16)$.

We also evaluated the sensitivities of the different tests based on tuberculosis localization. In Table 2, sensitivities for pulmonary, extra-pulmonary and disseminated tuberculosis (pulmonary and extra-pulmonary tuberculosis) are shown. Considering each test per se, no significant difference in proportion of positive results was observed in patients with active tuberculosis according to tuberculosis localization [with the exception of TST for which the highest proportion of positive results was recorded among patients with pulmonary tuberculosis $(p=0.027)]$.

Of the 132 patients with culture confirmed pulmonary tuberculosis, 28 had a negative sputum smear. Among these patients, sensitivity results were $64.3 \%$ (18/28; CI, 44.1-81.4) for RD1 selected peptides test, $84.6 \%$ (22/26; CI, 65.1-95.6) for TST, 88.0\% (22/25; CI, 68.8-97.5) for QuantiFERON-TB GOLD InTube, and $83.3 \%$ (5/6; CI, 35.9-99.6) for T-SPOT.TB. Compared to RD1 selected peptides test, the sensitivity for active tuberculosis was significantly higher only for QuantiFERON-TB GOLD In-Tube $(p=0.037)$. M. tuberculosis-specific RNA amplification was performed in 22 of these 27 subjects (8 sputa and 16 broncholavage) and resulted positive in $75 \%$ of sputa $(6 / 8$; CI, 34.9-96.8) and in 93.8\% of broncholavages (15/16; CI, 69.8-99.8) with an overall sensitivity of $86.4 \%$ (19/22; CI, 65.1-97.1). Among the 118 patients with smear positive culture confirmed pulmonary tuberculosis the sensitivity results were $77.1 \%$ (91/118; CI, 68.584.3) by RD1 selected peptides test, 87.6\% (78/89; CI, 79.0-93.7) by TST, $80.5 \%(66 / 82$; CI, 70.3-88.4) by QuantiFERON-TB GOLD In-Tube, and $88.0 \%$ (44/50; CI, 75.7-95.5) by TSPOT.TB. No statistical difference was found between the single tests' results obtained in those smear positive vs those smear negative.

Specificity for active tuberculosis was 70.6\% (CI, 63.7-76.8\%) with RD1 selected peptides test, 48.0\% (CI, 39.8-56.3\%) with TST, $61.9 \%$ (CI, 51.4-71.5\%) with QuantiFERON-TB GOLD In-Tube, and 44.3\% (CI, 34.2-54.8\%) with T-SPOT.TB (Table 2). The specificity for active tuberculosis was significantly higher for the assay based on RDl selected peptides compared with TST $(\mathrm{p}<0.001)$ and T-SPOT.TB $(\mathrm{p}<0.001)$.

Then we assessed whether the pair-wise combination of the tests could lead to a better evaluation of active tuberculosis diagnosis and we calculated the probabilities for the potential outcomes (double positive, double negative and discordant results) given the disease status. 
The probability of a positive result of at least one of the two tests considered was higher than sensitivities of each test (Table 3). In particular the assay based on RD1 selected peptides combined with TST led to a sensitivity of $92.4 \%$ (CI, 87.3-96.0\%), with QuantiFERON-TB GOLD In-Tube of $85.7 \%$ (CI, 79.2-90.8\%), and with T-SPOT.TB of 88.6\% (CI, 80.1-94.4\%) (Table 3). To note that the highest sensitivities were obtained by using the combination of TST with either QuantiFERON-TB GOLD InTube [sensitivity of $97.7 \%$ (CI, 93.0-99.5\%)], or T-SPOT.TB [sensitivity of $97.1 \%$ (CI, 89.9-94.4\%) (Table 3)]. Then we estimated the likelihood ratios for the combination of the tests. Positive results from both tests provided likelihood ratios of 2.92 for the RD1 selected peptides test combined with TST [CI, 2.153.98], 3.21 with QuantiFERON-TB GOLD In-tube, [CI, 2.114.89], and 2.20 with T-SPOT.TB [CI, 1.59-3.07], (Table 4).

Negative results on combined tests were associated with lower negative likelihood ratios compared to that obtained by single assay especially when a blood test was associated with TST. In particular the negative likelihood ratio of the combination of RD1 selected peptides test with TST was 0.19 (CI, 0.11-0.33), with QuantiFERON-TB GOLD In-Tube was 0.25 (CI, 0.16-0.38) and with T-SPOT. TB was 0.27 (CI, 0.14-0.50) (Table 4). To note that better negative likelihood ratios were obtained by the combination of commercial tests with TST (Table 4).

\section{Discussion}

We present the results of a prospective multicenter trial of the TBNET that was designed to investigate the performance of a novel blood test based on RD1 selected peptides for the immunodiagnosis of active tuberculosis.

The novel assay had a higher specificity for active tuberculosis than the TST and commercial IGRAs, but it had a lower sensitivity. Although the novel assay had a higher likelihood ratio, none of the tests evaluated was accurate enough to discriminate patients with active tuberculosis from those without, probably because of the high levels of LTBI in the population studied. Combined use of TST with either the RD1 selected peptides test or with the other commercial IGRAs improved the diagnostic accuracy for active disease, especially when considering the combination of negative results, contributing to rapid exclusion of tuberculosis. However, $M$. tuberculosis culture remains the diagnostic gold standard for active tuberculosis and is required for identifying drug resistance. Consequently, active tuberculosis should not be ruled out in a high-risk individual without a thorough microbiological work-up for tuberculosis disease.

The specificity of the assay based on RD1 selected peptides was lower in this multicenter trial compared with earlier, smaller studies of more limited patient groups $[15,16]$. Nevertheless, also in the present study the test based on RD1 selected peptides maintains the higher specificity compared to commercial IGRAs and TST which is not unexpected. In fact, the commercial IGRAs and TST use a greater variety of epitopes to elicit $M$. tuberculosis immune responses by effector memory T-cells [28-29] being TST a crude preparation of several mycobacterial antigens, and commercially IGRAs based on pools of overlapping peptides spanning the whole length of CFP10 and ESAT-6 proteins [5]. Conversely the selective approach of the design of the test based on RD1 selected peptides reduces false positive test results at the cost of a loss of diagnostic sensitivity [1416]. Which would be more acceptable between false positive test results that may lead to overtreatment or false negative test results that potentially lead to missing of cases with active tuberculosis to be treated, is a matter of debate and is largely dependent upon the prevalence of $M$. tuberculosis infection and the pre-test probability of tuberculosis in a community.

Specificity of commercial IGRAs is considerably lower in the present study compared to what was reported in a recent updated meta-analysis [30]. This may be due to the fact that this report involve the enrollment of patients with a suspicion of active tuberculosis that could by affected also by LTBI, while the literature reported in the meta-analyses [30] enclosed low-risk subjects with no known tuberculosis exposure in low incidence settings. Conversely sensitivity results were similar to those recently reported in the literature because based on patients with active disease $[5,16,30]$.

In terms of parameters used to evaluate the accuracy of diagnostic tests it is important to consider that while sensitivity and specificity are easy and straightforward measures, they are limited and must be considered as surrogates for patient-important outcomes. There is still lack of adequate data on important outcomes such as accuracy of diagnostic algorithms (rather than single tests), incremental or added value of IGRAs, impact of IGRAs on clinical decisionmaking and therapeutic choices, and the prognostic ability of IGRAs to accurately identify individuals with LTBI who are at the highest risk for progressing to active tuberculosis and therefore are most likely to benefit from preventive therapy. These issues need to be evaluated for further studies.

New tools for a rapid diagnosis of active tuberculosis are needed especially for the smear negative and extra-pulmonary cases. In the smear negative tuberculosis group, the overall response to RD1 selected peptides was $64.3 \%$ and, among the tests used for comparison, a significantly higher sensitivity was found only for the QuantiFERON-TB GOLD In-Tube. Conversely the sensitivity for extra-pulmonary tuberculosis was $71.4 \%$ for the RDl selected peptides and this value did not significantly differ from the results obtained by the other tests, with the exception of TST. However, in general, the size of these sub-groups of patients was small and no definitive conclusion can be drawn.

The rate of indeterminate results in our study was similar to the $3 \%$ to $4 \%$ rates observed in other studies [31-33]. Moreover, the

Table 3. Estimates of sensitivities for the combination of the diagnostic tests studied.

\begin{tabular}{|c|c|c|c|}
\hline & \multicolumn{3}{|c|}{ Combined tests \% [CI] } \\
\hline & TST & QuantiFERON-TB GOLD In-Tube & T-SPOT.TB \\
\hline RD1 selected peptides test & $92.4[87.3-96.0]$ & $85.7[79.2-90.8]$ & $88.6[80.1-94.4]$ \\
\hline TST & - & 97.7 [93.0-99.5] & 97.1 [89.9-99.6] \\
\hline QuantiFERON-TB GOLD In-Tube & - & - & $87.0[76.7-93.9]$ \\
\hline
\end{tabular}


Table 4. Estimates of likelihood ratios for the combination of the diagnostic tests studied according to the disease status.

\begin{tabular}{|c|c|c|c|}
\hline Combined tests & $\begin{array}{l}\text { Subjects with } \\
\text { Active TB n (\%) }\end{array}$ & $\begin{array}{l}\text { Subjects without Active } \\
\text { TB n (\%) }\end{array}$ & $\begin{array}{l}\text { Combined likelihood } \\
\text { ratio }[\mathrm{Cl}]\end{array}$ \\
\hline RD1 test pos/TST pos & $116(68.1)$ & $35(23.3)$ & $2.92[2.15-3.98]$ \\
\hline RD1 test neg/TST neg & $13(7.7)$ & $60(40.0)$ & $0.19[0.11-0.33]$ \\
\hline RD1 test pos/TST neg & $11(6.5)$ & $12(8.00)$ & $0.81[0.37-1.78]$ \\
\hline RD1 test neg/TST pos & $30(17.7)$ & $43(28.7)$ & $0.62[0.41-0.93]$ \\
\hline Total & $170(100.0)$ & $150(100.0)$ & \\
\hline RD1 test pos/QuantiFERON pos & $99(64.3)$ & $19(20.0)$ & $3.21[2.11-4.89]$ \\
\hline RD1 test neg/ QuantiFERON neg & $22(14.3)$ & $55(57.8)$ & $0.25[0.16-0.38]$ \\
\hline RD1 test pos/ QuantiFERON neg & $12(7.8)$ & $3(3.2)$ & $2.47[0.71-8.52]$ \\
\hline RD1 test neg/ QuantiFERON pos & $21(13.6)$ & $18(19.0)$ & $0.72[0.40-1.28]$ \\
\hline Total & $154(100.0)$ & $95(100.0)$ & \\
\hline RD1 test pos/T-SPOT.TB pos & $60(68.1)$ & $30(30.9)$ & $2.20[1.59-3.07]$ \\
\hline RD1 test neg/T-SPOT.TB neg & $10(11.4)$ & $41(42.3)$ & $0.27[0.14-0.50]$ \\
\hline RD1 test pos/T-SPOT.TB neg & $3(3.4)$ & $2(2.1)$ & $1.65[0.28-9.67]$ \\
\hline RD1 test neg/T-SPOT.TB pos & $15(17.1)$ & $24(24.7)$ & $0.69[0.39-1.23]$ \\
\hline Total & $88(100.0)$ & $97(100.0)$ & \\
\hline QuantiFERON pos/TST pos & $81(65.8)$ & $23(30.7)$ & 2.15 [1.49-3.09] \\
\hline QuantiFERON neg/TST neg & $3(2.4)$ & $27(36.0)$ & $0.07[0.02-0.22]$ \\
\hline QuantiFERON pos/TST neg & $19(15.5)$ & $9(12.0)$ & $1.29[0.61-2.70]$ \\
\hline QuantiFERON neg/TST pos & $20(16.3)$ & $16(21.3)$ & $0.76[0.42-1.38]$ \\
\hline Total & $123(100.0)$ & $75(100.0)$ & \\
\hline QuantiFERON pos/T-SPOT.TB pos & $50(72.5)$ & $20(41.7)$ & $1.74[1.21-2.51]$ \\
\hline QuantiFERON neg/T-SPOT.TB neg & $9(13.0)$ & $24(50.0)$ & $0.26[0.13-0.51]$ \\
\hline QuantiFERON pos/T-SPOT.TB neg & $2(2.9)$ & $0(0.0)$ & NA \\
\hline QuantiFERON neg/T-SPOT.TB pos & $8(11.6)$ & $4(8.3)$ & $1.39[0.44-4.36]$ \\
\hline Total & $69(100.0)$ & $48(100.0)$ & \\
\hline TST pos/T-SPOT.TB pos & $50(72.4)$ & $29(36.7)$ & $1.97[1.43-2.73]$ \\
\hline TST neg/T-SPOT.TB neg & $2(2.9)$ & $23(29.1)$ & $0.10[0.02-0.41]$ \\
\hline TST pos/T-SPOT.TB neg & $5(7.3)$ & $10(12.7)$ & $0.57[0.21-1.59]$ \\
\hline TST neg/T-SPOT.TB pos & $12(17.4)$ & $17(21.5)$ & $0.81[0.42-1.57]$ \\
\hline Total & $69(100.0)$ & $79(100.0)$ & \\
\hline
\end{tabular}

Abbreviations:

TB: tuberculosis; RD: region of difference; RD1 test: test based on the RD1 selected peptides; TST: tuberculin skin test; Cl: confidence interval; pos: positive; neg: negative; QuantiFERON: QuantiFERON-TB GOLD In-Tube.

doi:10.1371/journal.pone.0003417.t004

proportion of patients with tuberculosis was no higher among those with indeterminate results. As expected, false-negative results to any of the immune assays considered were associated with factors known to cause anergy such as disseminated disease.

Taken together, our results suggest that none of the tests considered is accurate enough to be used in clinical practice to diagnose active tuberculosis, and new approaches should be considered. Recently, it has been shown that the discrimination of active tuberculosis from LTBI may be ameliorated by documenting recruitment of $M$. tuberculosis-specific lymphocytes to the site of the infection by RD1-specific ELISPOT assays [34-36] which may open a new strategy for the distinction of the two different status of tuberculosis.

Another recent study suggests that the combination of different immunodiagnostic tests may improve their diagnostic accuracy [21]. In fact it has been shown that, T-SPOT.TB or a new ELISPOT assay incorporating the Rv3879c to RDl antigens, when used in combination with TST have an increased positive and negative likelihood ratio compared with single tests suggesting that this approach can be used to exclude active tuberculosis in patients with moderate to high pre-test probability of disease [21]. The results of the present study confirm and extend these findings. In fact, we confirmed the diagnostic performance of the commercial version of T-SPOT.TB when combined to TST, and additionally, we substantiated the data analyzing the results obtained by the assay based on RD1 selected peptides and the QuantiFERON-TB GOLD In-Tube. The likelihood ratio of a negative test result that was 0.38 with the assay based on RD1 selected peptides, became 0.19 when combined with a negative TST. Similarly, the combination of a negative result of the QuantiFERON-TB GOLD In-Tube assay with a negative TST scoring reached the lowest negative likelihood ratio of 0.07 . This means that while a negative result to RD1 selected peptides test reduces 2.6-fold the odds of tuberculosis, a negative result to both tests, RD1 selected peptides and TST, would reduce the odds of tuberculosis 5.3-fold and using the combination of TST and 
QuantiFERON-TB GOLD In-Tube by 14.2-fold which may currently be the best available option to rapidly exclude tuberculosis by immunodiagnostic tests. Conversely, a positive result of the assay based on RDl selected peptides with a corresponding likelihood ratio of 2.48 is of limited value and it is not significantly modified in those with positive results from either the assays based on RDl selected peptides or the TST or QuantiFERON-TB GOLD In-Tube (likelihood ratio goes up to 2.92 and 3.21 respectively). Similarly, results on other tests combinations improve the positive likelihood ratio of the single test per se, but do not increase significantly that obtained by the combination of RD1 selected peptides with TST or QuantiFERON-TB GOLD In-Tube.

The higher sensitivity of combined use of the novel assay or commercial IGRAs with TST reflects the fact that patients who had a false-negative result with one test were distinct from those who had a false-negative result with the other. This implies that distinct immunologic processes underlie failure of these different, yet complementary, immune-based tests.

The study has some limitations. Not all individuals were tested by all the assays in parallel, as not all techniques for the different tests were established in the participating centers. In addition, the restricted number of immunocompromised patients does not allow a generalization of the results to this patients group. However, the prospective and multicenter design of the study, the high consistency of data across the different centers and the large

\section{References}

1. (2000) Targeted tuberculin testing and treatment of latent tuberculosis infection. Am J Respir Crit Care Med 161: S221-247.

2. Smieja MJ, Marchetti CA, Cook DJ, Smaill FM (2000) Isoniazid for preventing tuberculosis in non-HIV infected persons. Cochrane Database Syst Rev. CD001363.

3. (2008) WHO. Global tuberculosis control: surveillance, planning, financing. In: WHO r, ed. Geneva, . pp 1-294.

4. Dinnes J, Deeks J, Kunst H, Gibson A, Cummins E, et al. (2007) A systematic review of rapid diagnostic tests for the detection of tuberculosis infection. Health Technol Assess 11: 1-196.

5. Pai M, Riley LW, Colford JM Jr (2004) Interferon-gamma assays in the immunodiagnosis of tuberculosis: a systematic review. Lancet Infect Dis 4: 761-76.

6. Richeldi L (2006) An update on the diagnosis of tuberculosis infection. Am J Respir Crit Care Med 174: 736-42.

7. Menzies D, Pai M, Comstock G (2007) Meta-analysis: new tests for the diagnosis of latent tuberculosis infection: areas of uncertainty and recommendations for research. Ann Intern Med 146: 340-54.

8. Lalvani A (2007) Diagnosing tuberculosis infection in the 21st century: new tools to tackle an old enemy. Chest 131: 1898-906.

9. Domínguez J, Ruiz-Manzano J, De Souza-Galvão M, Latorre I, Milà C, Blanco S, et al. (2008) Comparison of two commercially available gamma interferon blood tests for immunodiagnosis of tuberculosis. Clin Vaccine Immunol 15: 168-71.

10. Arend SM, Thijsen SF, Leyten EM, Bouwman JJ, Franken WP, Koster BF, et al. (2007) Comparison of two interferon-gamma assays and tuberculin skin test for tracing tuberculosis contacts. Am J Respir Crit Care Med 175: 618-27.

11. Lalvani A, Pathan AA, Durkan H, Wilkinson KA, Whelan A, Deeks JJ, et al. (2001) Enhanced contact tracing and spatial tracking of Mycobacterium tuberculosis infection by enumeration of antigen-specific T cells. Lancet 357: 2017-21.

12. Lalvani A, Pathan AA, McShane H, Wilkinson RJ, Latif M, Conlon CP, et al. (2001) Rapid detection of Mycobacterium tuberculosis infection by enumeration of antigen-specific T cells. Am J Respir Crit Care Med 163: 824-8.

13. Ewer K, Deeks J, Alvarez L, Bryant G, Waller S, Andersen P, et al. (2003) Comparison of T-cell-based assay with tuberculin skin test for diagnosis of Mycobacterium tuberculosis infection in a school tuberculosis outbreak. Lancet 361: 1168-73.

14. Vincenti D, Carrara S, De Mori P, Pucillo LP, Petrosillo N, et al. (2003) Identification of early secretory antigen target- 6 epitopes for the immunodiagnosis of active tuberculosis. Mol Med 2003 9: 105-11.

15. Goletti D, Vincenti D, Carrara S, Butera O, Bizzoni F, et al. (2005) Selected RD1 peptides for active tuberculosis diagnosis: comparison of a gamma interferon whole-blood enzyme-linked immunosorbent assay and an enzymelinked immunospot assay. Clin Diagn Lab Immunol 12: 1311-6.

16. Goletti D, Carrara S, Vincenti D, Saltini C, Rizzi EB, et al. (2006) Accuracy of an immune diagnostic assay based on RDl selected epitopes for active tuberculosis in a clinical setting: a pilot study. Clin Microbiol Infect 12: 544-50. number of patients enrolled to evaluate the diagnostic accuracy of different immune based tests in clinical practice render the results robust.

In conclusion, current approaches to elicit $M$. tuberculosis-specific immune responses in PBMC or in the skin by using either a broad or narrow spectrum of epitopes of RD1 mycobacterial antigens have a limited value for the diagnosis of active tuberculosis, as these tests do not reliably distinguish patients with active tuberculosis from those without. This is important to be considered in populations with a high pre-test probability of $M$. tuberculosis infection. However, the combined use of negative test results obtained by IGRAs or the test based on RDl selected peptides with TST may enable rapid exclusion of tuberculosis.

\section{Acknowledgments}

The authors are grateful to all patients and nursing staff who took part in this study. The authors thank Dr Carla Nisii for the precious editorial support.

\section{Author Contributions}

Conceived and designed the experiments: DG GBM CL EG. Performed the experiments: OB ME IS EB RHD JD IL. Analyzed the data: DG SC VV DMC RKM CA AN EG. Contributed reagents/materials/analysis tools: DG MA VV DMC RKM NP FNL GI. Wrote the paper: DG SC CA GBM CL EG.

17. Goletti D, Parracino MP, Butera O, Bizzoni F, Casetti R, et al. (2007) Isoniazid prophylaxis differently modulates T-cell responses to RD1-epitopes in contacts recently exposed to Mycobacterium tuberculosis: a pilot study. Respir Res 8: 5.

18. Goletti D, Butera O, Bizzoni F, Casetti R, Girardi E, et al. (2006) Region of difference 1 antigen-specific CD4+ memory $\mathrm{T}$ cells correlate with a favorable outcome of tuberculosis. J Infect Dis 194: 984-92.

19. Carrara S, Vincenti D, Petrosillo N, Amicosante M, Girardi E, et al. (2004) Use of a $\mathrm{T}$ cell-based assay for monitoring efficacy of antituberculosis therapy. Clin Infect Dis 38: 754-6.

20. http://www.ministerosalute.it/promozione/malattie/bollettino.jsp, accessed July 30 th, 2008.

21. Dosanjh DP, Hinks TS, Innes JA, Deeks JJ, Pasvol G, et al. (2008) Improved diagnostic evaluation of suspected tuberculosis. Ann Intern Med 148: 325-336.

22. Migliori GB, Borghesi A, Spanevello A, Eriki P, Raviglione M, et al. (1994) Risk of infection and estimated incidence of tuberculosis in northern Uganda. Eur Respir J 7: 946-53.

23. Ten Dam HG (1985) Surveillance of tuberculosis by means of tuberculin surveys. World health Organization, Geneva, Switzerland, 1985. WHO/TB/85 145: 1-17.

24. (2000) American Thoracic Society. Diagnostic standards and classification of tuberculosis in adults and children. Am J Respir Crit Care Med 161: 1376 95.

25. (2000) Control and prevention of tuberculosis in the United Kingdom: code of practice 2000. Thorax 55: 887-901.

26. Wang L, Turner MO, Elwood RK, Schulzer M, FitzGerald JM (2002) A metaanalysis of the effect of Bacille Calmette Guérin vaccination on tuberculin skin test measurements. Thorax 57: 804-9.

27. Scarpellini P, Tasca S, Galli L, Beretta A, Lazzarin A, Fortis C (2004) Selected pool of peptides from ESAT-6 and CFP-10 proteins for detection of Mycobacterium tuberculosis infection. J Clin Microb 42: 3469-74.

28. Streitz M, Tesfa L, Yildirim V, Yahyazadeh A, Ulrichs T, et al. (2007) Loss of receptor on tuberculin-reactive T-cells marks active pulmonary tuberculosis. PLOS ONE 15:2: e735.

29. Picker LJ, Treer JR, Ferguson-Darnell B, Collins PA, Bergstresser PR, et al. (1993) Control of lymphocyte recirculation in man. II. Differential regulation of the cutaneous lymphocyte-associated antigen, a tissue-selective homing receptor for skin-homing T cells. J Immunol 150: 1122-1136.

30. Pai M, Zwerling A, Menzies D (2008) Systematic review: T-cell-based assays for the diagnosis of latent tuberculosis infection: an update. Ann Intern Med 5:149: 177-84.

31. Ferrara G, Losi M, D'Amico R, Roversi P, Piro R, et al. (2006) Use in routine clinical practice of two commercial blood tests for diagnosis of infection with Mycobacterium tuberculosis: a prospective study. Lancet 367: 1328-34.

32. Piana F, Codecasa LR, Cavallerio P, Ferrarese M, Migliori GB, et al. (2006) Use of a T-cell-based test for detection of tuberculosis infection among immunocompromised patients. Eur Respir J 28: 31-4. 
33. Ferrara G, Losi M, Meacci M, Meccugni B, Piro R, Roversi P, et al. (2005) Routine hospital use of a new commercial whole blood interferon-gamma assay for the diagnosis of tuberculosis infection. Am J Respir Crit Care Med 172: 631-5.

34. Jafari C, Ernst M, Strassburg A, Greinert U, Kalsdorf B, et al. (2008) Local immunodiagnosis of pulmonary tuberculosis by enzyme-linked immunospot. Eur Respir J 31: 261-5.
35. Jafari C, Ernst M, Kalsdorf B, Greinert U, Diel R, Kirsten D, et al. (2006) Rapid diagnosis of smear-negative tuberculosis by bronchoalveolar lavage enzymelinked immunospot. Am J Respir Crit Care Med 174: 1048-54.

36. Wilkinson KA, Wilkinson RJ, Pathan A, Ewer K, Prakash M, et al. (2005) Ex vivo characterization of early secretory antigenic target 6 -specific T cells at sites of active disease in pleural tuberculosis. Clin Infect Dis 40: 184-7. 